\title{
(C) OPEN ACCESS \\ Ultrasonic measurement of optic nerve sheath diameter: a non-invasive surrogate approach for dynamic, real-time evaluation of intracranial pressure
}

\author{
Li-min Chen, Li-juan Wang, Yang Hu, Xiao-han Jiang, Yu-zhi Wang, Ying-qi Xing
}

Department of Neurology and Neuroscience Center, The First Hospital of Jilin University, Changchun, China

\section{Correspondence to}

Ying-qi Xing, The First Hospital of Jilin University, Changchun 130021, China; xingyq2009@ sina.com

L-mC and L-jW contributed equally.

Received 22 July 2018 Revised 9 October 2018 Accepted 13 October 2018 Published Online First 25 October 2018
Check for updates

(C) Author(s) (or their employer(s)) 2019. Re-use permitted under CC BY-NC. No commercial re-use. See rights and permissions. Published by BMJ.

To cite: Chen $\mathrm{L}$, Wang $\mathrm{L}$, Hu Y, et al. Br J Ophthalmol 2019:103:437-441.

\section{ABSTRACT}

The current study aimed to identify whether ultrasonographic measurements of optic nerve sheath diameter (ONSD) could dynamically and sensitively evaluate real-time intracranial pressure (ICP). ONSD measurements were performed approximately $5 \mathrm{~min}$ prior to and after a lumbar puncture (LP). A total of 84 patients (mean \pm SD age, $43.5 \pm 14.7$ years; 41 (49\%) men; 18 patients with elevated ICP) were included in the study. The Spearman correlation coefficients between the two observers were 0.779 and 0.703 in the transverse section and 0.751 and 0.788 in the vertical section for the left and right eyes, respectively. The median (IQR) change in ONSD $(\triangle \mathrm{ONSD})$ and change in ICP $(\triangle \mathrm{ICP})$ were $0.11(0.05-0.21) \mathrm{mm}$ and $30(20-40) \mathrm{mmH}_{2} \mathrm{O}$, respectively, for all participants. With a reduction in cerebrospinal fluid pressure, 80 subjects (95\%) showed an immediate drop in ONSD; the median (IQR) decreased from $4.13(4.02-4.38) \mathrm{mm}$ to $4.02(3.90-4.23) \mathrm{mm}$ $(p<0.001)$. Significant correlations were found between ONSD and ICP before $\operatorname{Lss}(r=0.482, p<0.01)$ and between $\triangle O N S D$ and $\triangle I C P(r=0.451, p<0.01)$. Ultrasonic measurement of ONSD can reflect the relative real-time changes in ICP.

\section{INTRODUCTION}

Detection of intracranial pressure (ICP) has become an important component in the clinical setting for the diagnosis and management of several neurological pathologies, such as head injury, hydrocephalus, subarachnoid haemorrhage and intracranial haematoma. ${ }^{12}$ Until recently, invasive ICP monitoring was the gold standard. ${ }^{3}$ Invasive techniques, although accurate and highly sensitive, require strict neurosurgical settings and cause side effects that must be managed, such as haemorrhage, infection and brain lesions. ${ }^{45}$ Therefore, it is necessary to explore non-invasive monitoring methods.

Papilloedema, a condition in which part of the optic nerve inside the eye swells due to increased pressure in or around the brain, has often been used for screening suspected increased ICP, but optic disc swelling in cases of raised ICP takes time. Therefore, this technique is not applied in emergency conditions or when an acute increase in ICP is suspected. ${ }^{36}$ Some non-invasive methods for measuring ICP are available. ${ }^{6-9}$ The instrument developed by Ragauskas $e t \mathrm{al}^{8}$ can directly assess the ICP. The measurement of optic nerve subarachnoid space can indirectly evaluate the ICP. ${ }^{9}$ Recently, studies have reported that measuring the optic nerve sheath diameter (ONSD) using non-invasive imaging technologies provides alternative markers for early elevated ICP. ${ }^{10-13}$ However, CT scans and MRI for ONSD measurements are time-consuming, costly and require patient transportation. Thus, ultrasound assessment of ONSD could be a better option because of its low cost and rapid bedside operation, especially for cases that are not suitable for the intensive care unit and require real-time monitoring of ICP. ${ }^{13}{ }^{14}$ Ultrasonic measurements of the ONSD have established different normal values for humans and determined the ONSD cut-off point for evaluating high ICP. ${ }^{15-17}$ Furthermore, we have derived a mathematical function to quantitatively assess ICP. ${ }^{18}$ However, it remains unclear whether the acute reduction in ICP may lead to an immediate effect on ONSD. Thus, we performed this study to investigate whether ultrasonographic measurements of ONSD could dynamically and sensitively evaluate real-time ICP.

\section{MATERIALS AND METHODS \\ Study population}

All patients who required lumbar punctures (LPs) for diagnostic purposes between August 2016 and January 2017 at the Department of Neurology were recruited. We collected data from consenting patients willing to participate in the study. The exclusion criteria were as follows: (1) age $<18$ years or $>80$ years; (2) eye or orbit diseases, such as glaucoma, lens opacity or trauma; and (3) ICP $<70$ $\mathrm{mmH}_{2} \mathrm{O}$ (if the ICP is too low, we cannot extract an equivalent amount of cerebrospinal fluid (CSF)). We collected detailed data from all the participants: age, sex, body mass index, waistline, head circumference, systolic blood pressure (SBP), diastolic blood pressure (DBP) and mean arterial blood pressure (calculated as $1 / 3 \times \mathrm{SBP}+2 / 3 \times \mathrm{DBP})$.

\section{Measurements}

Optic nerve sheath diameter

ONSD measurements were performed approximately 5 min prior to and after LP (figure 1). The operations were performed by two independent and experienced operators who were blind to each other's results. The ONSD was measured with the Delica MVU-6300 (Shenzhen, Guangzhou, China), using a $14-5 \mathrm{MHz}$ probe and B-mode. The patients were examined in a supine relaxed position. The probe was gently placed on the closed upper eyelid with a standard ultrasound gel and adjusted to a suitable angle to display the optic nerve entry into 


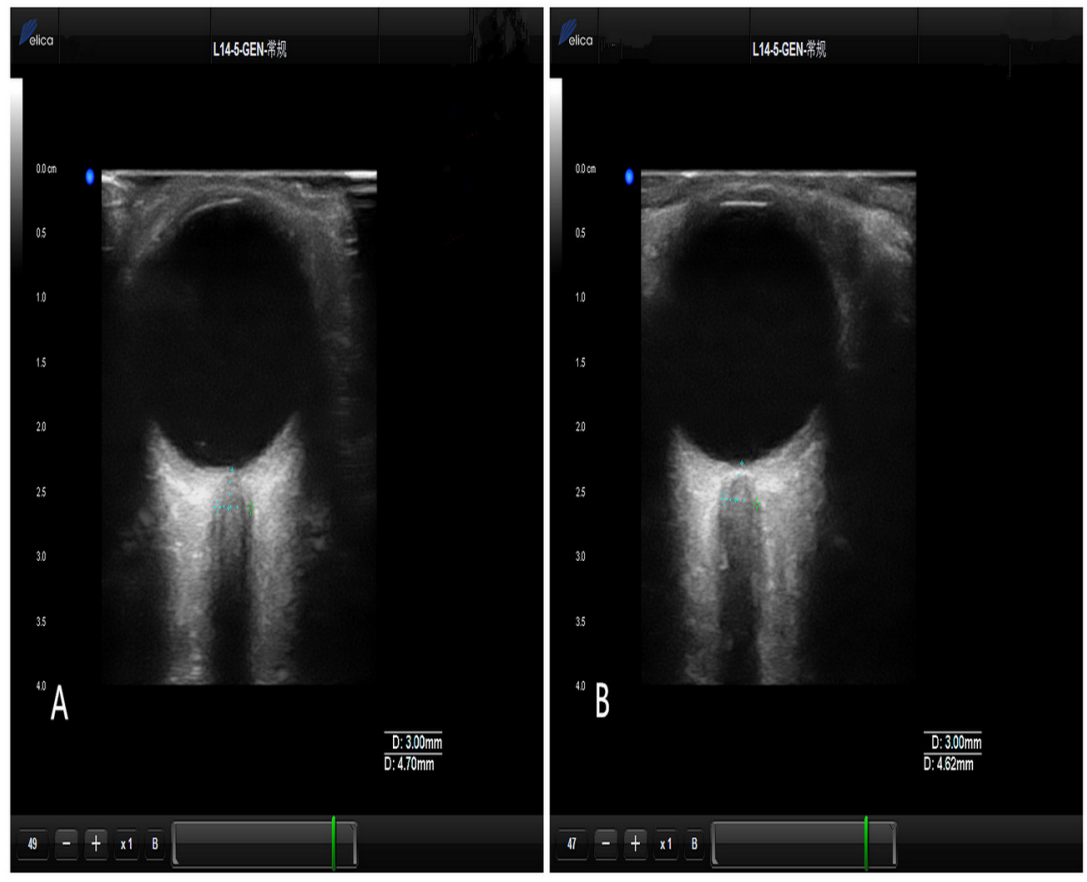

Figure 1 ONSD measurements: (A) ONSD before the LP; (B) ONSD after the LP. LP, lumbar puncture; ONSD, optic nerve sheath diameter.

the eyeball. According to the ALARA ('as low as reasonably achievable') principle, the output of the ultrasonic instrumentation was adjusted to the requirements of orbital sonography. ${ }^{19}$ As recommended, ONSD measurements were performed at a depth of $3 \mathrm{~mm}$ behind the eye globe, ${ }^{13}$ and two measurements were performed for each eye. The first measurement was performed in the transverse section and the second was performed in the vertical section, and thus a total of eight values were obtained. The final ONSD measurement value for every patient was derived from the average of the eight values to reduce deviation. Operator variability has been confirmed to be minimised using the aforementioned approaches. ${ }^{2021}$

\section{Lumbar puncture}

The LP was performed by a senior neurologist according to a standard procedure where the ICP was recorded in $\mathrm{mmH}_{2} \mathrm{O}$. Initial and final ICP values were recorded. Elevated ICP was defined as a pressure $>200 \mathrm{mmH}_{2} \mathrm{O}^{22}$ Additionally, 3-4 mL of CSF was collected for laboratory examination.

\section{Statistical analyses}

Descriptive analysis was performed for qualitative data using frequency and percentage; for quantitative data consistent with the normal distribution, the mean and SD were used for analysis; for data that did not meet the criteria for a normal distribution, analysis involved the median with IQR. Normality of the distribution was assessed using histograms and the Kolmogorov-Smirnov test. Spearman correlation analysis was performed to compare the measurements between two eyes and two observers. A Bland-Altman analysis was performed to determine interobserver reliability. The Wilcoxon rank-sum test was used to compare the differences in the ONSD before and after LP. The correlation between the ONSD and ICP was evaluated using Spearman correlation analysis. Changes in the ONSD ( $\triangle$ ONSD) and ICP $(\triangle \mathrm{ICP})$ before and after LP were assessed. The correlation between $\triangle$ ONSD and $\triangle \mathrm{ICP}$ was evaluated using Spearman correlation analysis. Statistical analyses were performed on a personal computer using SPSS V.20.0 software, and $\mathrm{p}<0.05$ was considered statistically significant.

\section{RESULTS}

A total of 84 patients (mean (SD) age, 43.5 (14.7) years; 41 (49\%) men; 18 patients with elevated ICP) were included in this study (table 1 ).

The Spearman correlation coefficient for the left eye between the two observers was 0.779 in the transverse section and 0.751 in the vertical section; for the right eye, it was 0.703 in the transverse section and 0.788 in the vertical section. A Bland-Altman analysis yielded a mean (SD) difference of $0.038(0.232) \mathrm{mm}$ in measurements between the two observers. Limits of agreement $($ mean $\pm 1.96 \times \mathrm{SD})$ were 0.493 and $-0.417 \mathrm{~mm}$. The ONSD values for all participants, measured before and after LP, ranged from 3.57 to $5.63 \mathrm{~mm}$, and from 3.26 to $5.01 \mathrm{~mm}$, respectively. The initial ICP for all participants ranged from 90 to 400 $\mathrm{mmH}_{2} \mathrm{O}$, and the final ICP ranged from 70 to $240 \mathrm{mmH}_{2} \mathrm{O}$. Variation ranges for different groups are listed in table 2.

The $\triangle \mathrm{ONSD}$ and $\triangle \mathrm{ICP}$ of the 84 patients ranged from 0 to $0.62 \mathrm{~mm}$ and from 0 to $180 \mathrm{mmH}_{2} \mathrm{O}$, respectively. The median (IQR) $\triangle$ ONSD and $\triangle \mathrm{ICP}$ were $0.11(0.05-0.21) \mathrm{mm}$ and 30 (20-40) $\mathrm{mmH}_{2} \mathrm{O}$, respectively, for all participants. The median (IQR) $\triangle$ ONSD and $\triangle \mathrm{ICP}$ for the different groups are shown in table 3 .

\begin{tabular}{ll}
\hline Table 1 & \\
\hline Demographics $(\mathrm{n}=84)$ & \\
\hline Age, mean (SD), years & $43.5(14.7)$ \\
\hline Male, $\mathrm{n}(\%)$ & $41(49)$ \\
\hline Body mass index, mean (SD), $\mathrm{kg} / \mathrm{m}^{2}$ & $23.6(4.5)$ \\
\hline Waistline, mean (SD), cm & $80.5(11.2)$ \\
\hline Head circumference, mean (SD), cm & $55.5(1.8)$ \\
\hline Mean arterial blood pressure, mean (SD), $\mathrm{mm} \mathrm{Hg}$ & $97.1(17.6)$ \\
\hline
\end{tabular}


Table 2 Variation range for different groups

\begin{tabular}{llllll}
\hline & \multicolumn{2}{l}{ Elevated ICP $(\mathrm{n}=18)$} & & \multicolumn{2}{l}{ Normal ICP $(\mathrm{n}=66)$} \\
\cline { 2 - 3 } \cline { 5 - 5 } \cline { 5 - 5 } & Before LP & After LP & & Before LP & After LP \\
\hline ONSD range $(\mathrm{mm})$ & $4.02-5.63$ & $3.87-5.01$ & & $3.57-4.70$ & $3.26-4.61$ \\
ICP range $\left(\mathrm{mmH}_{2} \mathrm{O}\right)$ & $200-400$ & $110-240$ & & $90-195$ & $70-180$ \\
\hline
\end{tabular}

ICP, intracranial pressure; LP, lumbar puncture; ONSD, optic nerve sheath diameter.

Statistically significant correlations were found between ONSD and ICP before LP $(\mathrm{r}=0.482, \mathrm{p}<0.01)$ and between $\triangle$ ONSD and $\Delta \mathrm{ICP}(\mathrm{r}=0.451, \mathrm{p}<0.01)$. With a reduction in CSF pressure, 80 subjects (95\%) showed an immediate drop in ONSD; the median (IQR) decreased from $4.13(4.02-4.38) \mathrm{mm}$ to $4.02(3.90-4.23)$ $\mathrm{mm}(\mathrm{p}<0.001)$ (figure 2). Only four patients showed no significant changes in the ONSD before and after LP; figure 3 shows the $\triangle \mathrm{ONSD}$ and $\triangle \mathrm{ICP}$ for the elevated ICP groups. The largest change in ONSD value observed in this study was from 5.63 to $5.01 \mathrm{~mm}$, and the largest change in ICP was of $180 \mathrm{~mm} \mathrm{Hg}$.

\section{DISCUSSION}

This study demonstrates that the ONSD decreases immediately following a reduction in CSF pressure, thereby confirming that ONSD reacts to ICP in real time. Our results, therefore, suggest that the measurement of ONSD is potentially a non-invasive approach for dynamic, real-time monitoring of ICP changes, especially in the early stages. The optic nerve is a part of the central nervous system, and is surrounded by the dura, arachnoid and pia mater; the optic nerve sheath is the continuation of the dura mater, and the subarachnoid space contains CSF. Thus, any increase in the ICP in the subarachnoid space is transferred to the fluid in the optic nerve surrounding it. ${ }^{23}$ For some diseases, such as elevated ICP secondary to venous sinus thrombosis, LP has been used to determine whether the ICP has declined, and this procedure may need to be repeated several times. Therefore, it would benefit the patients considerably if an ONSD measurement could help determine the ICP. Several studies have confirmed that ONSD changes with ICP. ${ }^{12} 132425$

Sachita Shah et $a l^{26}$ investigated intraobserver and interobserver reliability among three emergency physicians using three different imaging protocols and suggested that comparable precision and interobserver reliability can be achieved. These findings are similar to those in our previous research. ${ }^{27}$ However, it remains unclear whether an acute reduction in ICP leads to an immediate effect on ONSD. Previous animal models ${ }^{28}$ and in vitro optic nerve experiments ${ }^{29} 30$ have focused on this problem. Our study is one of the few that evaluate alterations in ONSD as ICP changes, in real-time. In a study of 12 patients, Hansen and Helmke ${ }^{31}$ showed ONSD changes that exhibited covariance with the alteration of lumbar CSF pressure during infusion tests (Ringer's solution pumped in the lower lumbar intervertebral segments at adjustable constant flow rates ranging from 0.5 to $4.0 \mathrm{~mL} / \mathrm{min}$ ), and found that the average maximum difference in ONSD between baseline and peak pressure conditions was $1.8 \mathrm{~mm}$ (range: $0.7-3.1 \mathrm{~mm}$ ). In their study, the change in CSF

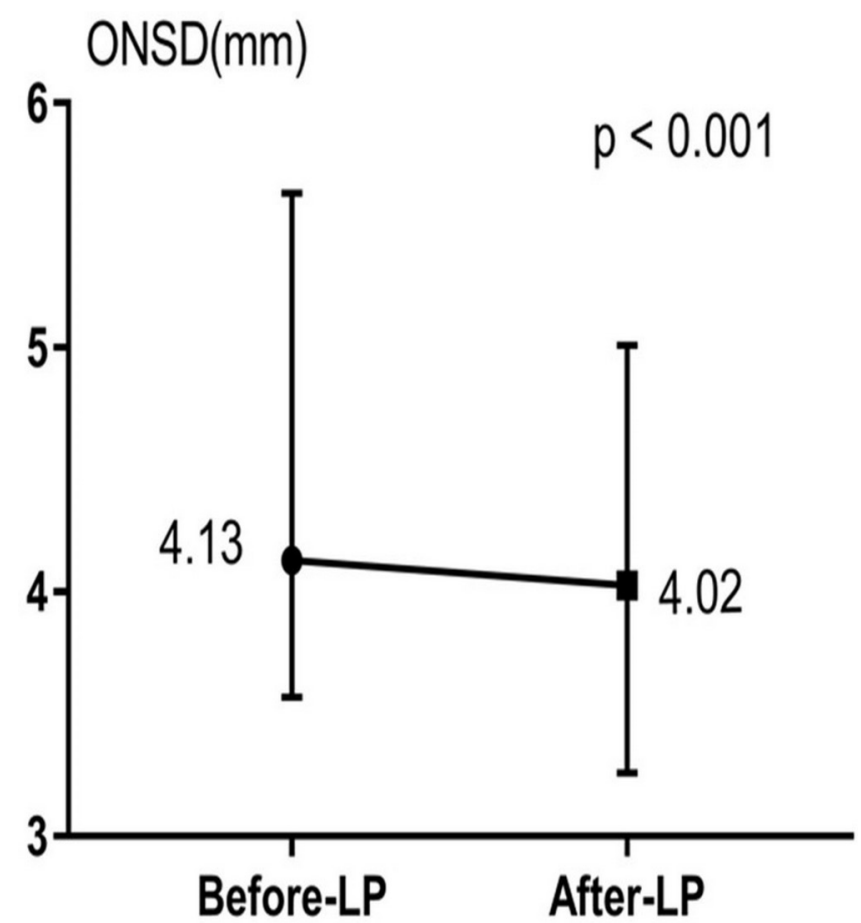

Figure 2 Median and range of optic nerve sheath diameter (ONSD) measured before and after lumbar puncture (LP) in all subjects.

pressure was controlled using infusion tests, and the range of increase in ICP was $17-51 \mathrm{~mm} \mathrm{Hg}\left(231.2-693.6 \mathrm{mmH}_{2} \mathrm{O}\right)$. When we measured the ONSD before and after LP, the range of reduction in $\triangle \mathrm{ICP}$ was from 0 to $180 \mathrm{mmH}_{2} \mathrm{O}$. We included 84 patients, and the final ONSD measurement value for every patient was derived from the average of the eight values obtained by two experienced operators to reduce deviation.

A prospective observational study demonstrated that the ONSD of children with hydrocephalus was significantly and immediately reduced after they underwent a ventriculo-peritoneal shunt surgery, which is similar to our results in adults. ${ }^{32}$ The difference is that the authors measured the ONSD $30 \mathrm{~min}$ after CSF drainage, while we performed the measurements within 5 min of the procedure. This study reflected the immediate, realtime changes of ONSD with changing ICP. The evaluation of ICP using an ONSD measurement has been widely used in clinical practice, and the standard operation is very important. Some reports ${ }^{15} 16$ have emphasised that ONSD measurements should be performed prior to the ICP monitoring device placement. Nevertheless, until now, there was no specific study to support this criterion. Our study shows that ONSD changes immediately after LP. Therefore, the measurements of ONSD should be performed before the operations that may affect the ICP. There could be two reasons why four patients in this study showed no changes in their ONSD after LP. The first reason is that changes in ICP lead to changes in the volume of the optic nerve sheath. Until now, studies have often used ultrasound to measure the

Table 3 Mean changes of $\triangle O N S D$ and $\triangle I C P$ in the different groups

\begin{tabular}{llll}
\hline & All subjects $(\mathrm{N}=84)$ & Normal ICP $(\mathbf{n}=66)$ & Elevated ICP $(\mathbf{n}=18)$ \\
\hline$\triangle \mathrm{ONSD}$, median (IQR), mm & $0.11(0.05-0.21)$ & $0.105(0.05-0.21)$ & $0.11(0.06-0.24)$ \\
$\triangle \mathrm{ICP}$, median (IQR), $\mathrm{mmH}_{2} \mathrm{O}$ & $30(20-40)$ & $30(20-40)$ & $30(20-82)$ \\
\hline
\end{tabular}

$\triangle \mathrm{ICP}$, the mean changes of intracranial pressure (ICP) before and after lumbar puncture.

$\triangle O N S D$, the mean changes in optic nerve sheath diameter (ONSD) values measured before and after lumbar puncture. 


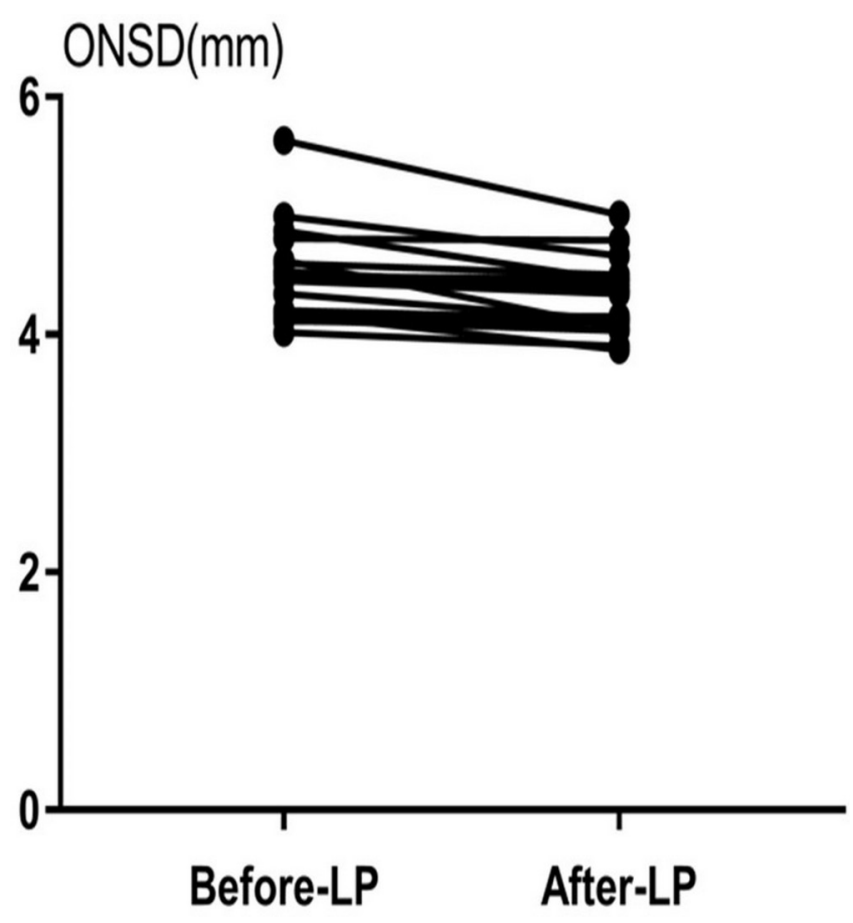

Figure 3 Change in optic nerve sheath diameter (ONSD) measured before and after lumbar puncture (LP) in subjects with elevated intracranial pressure.

diameter changes of the optic nerve sheath to reflect the volume changes of optic nerve sheath. However, one must consider that the optic nerve sheath may not be a perfectly circular structure. Killer et $a l^{23}$ studied 12 optic nerves from nine patients; the results showed that the subarachnoid space of the human optic nerve is not a homogeneous, empty chamber filled with CSF. Instead, it contains a complex arachnoid trabeculae and septa system to separate the subarachnoid space, which displays considerable numerical and structural changes according to their location in different portions of the optic nerve. Therefore, ONSD might vary from patient to patient and may not be the same in all locations of the sheath. To increase accuracy, we performed two cross-sectional scans to measure ONSD, in the transverse and vertical planes, and the average of the eight values represents the diameter changes of optic nerve sheath in our study. This may be crucial for cases where the diameter changes of optic nerve sheath do not perfectly reflect the volume changes of optic nerve sheath. The other possible reason for the lack of change in ONSD is that, although ICP is certainly an important factor, there are other factors that may affect the optic nerve sheath in vivo, and these factors need to be investigated in future studies.

Our study also has some limitations. First, this study was limited by its modest sample size. Thus, studies with larger samples should be conducted in the future. Second, the ICP measured using the LP manometer in this study has an upper limit of $400 \mathrm{mmH}_{2} \mathrm{O}$, so evaluating cases with even higher ICP in future studies would offer additional insights. Third, there are other non-invasive measurements for evaluating ICP by ultrasound that should be considered for investigation in our future research. Finally, the individual optic nerve differences should be taken into consideration in future research. Taken together, our results demonstrate that ultrasonic measurement of ONSD can reflect the relative real-time changes in ICP.
Acknowledgements The authors thank the patients who participated in this study and their relatives.

Contributors $Y-q X$ and L-jW conceived and designed the experiments. L-mC, X-hJ, $\mathrm{YH}$, and $\mathrm{Y}-\mathrm{zW}$ performed the experiments. L-mC analyzed the data and wrote the paper.

Funding This project was supported by the National Natural Science Foundation of China (no 8180070275).

Competing interests None declared.

Patient consent Obtained.

Ethics approval This study was approved by the ethics committee of The First Hospital of Jilin University (approval number: 2016-375).

Provenance and peer review Not commissioned; externally peer reviewed.

Open access This is an open access article distributed in accordance with the Creative Commons Attribution Non Commercial (CC BY-NC 4.0) license, which permits others to distribute, remix, adapt, build upon this work non-commercially, and license their derivative works on different terms, provided the original work is properly cited, appropriate credit is given, any changes made indicated, and the use is non-commercial. See: http://creativecommons.org/licenses/by-nc/4.0

\section{REFERENCES}

1 Balestreri M, Czosnyka M, Hutchinson P, et al. Impact of intracranial pressure and cerebral perfusion pressure on severe disability and mortality after head injury. Neurocrit Care 2006;4:008-13.

2 Czosnyka M, Pickard JD. Monitoring and interpretation of intracranial pressure. J Neurol Neurosurg Psychiatry 2004;75:813-21.

3 Raboel PH, Bartek J, Andresen M, et al. Intracranial pressure monitoring: invasive versus non-invasive methods-a review. Crit Care Res Pract 2012;2012:1-14.

4 Hagel S, Bruns T, Pletz MW, et al. External ventricular drain infections: risk factors and outcome. Interdiscip Perspect Infect Dis 2014;2014:1-6.

5 Dasic D, Hanna SJ, Bojanic S, et al. External ventricular drain infection: the effect of a strict protocol on infection rates and a review of the literature. $\mathrm{Br} J$ Neurosurg 2006;20:296-300.

6 Robba C, Bacigaluppi S, Cardim D, et al. Non-invasive assessment of intracranial pressure. Acta Neurol Scand 2016;134:4-21.

7 Hamarat Y, Deimantavicius M, Kalvaitis E, et al. Location of the internal carotid artery and ophthalmic artery segments for non-invasive intracranial pressure measurement by multi-depth TCD. Libyan J Med 2017;12:1384290.

8 Ragauskas A, Matijosaitis V, Zakelis R, et al. Clinical assessment of noninvasive intracranial pressure absolute value measurement method. Neurology 2012;78:1684-91.

9 Liu H, Yang D, Ma T, et al. Measurement and associations of the optic nerve subarachnoid space in normal tension and primary open-angle glaucoma. Am J Ophthalmol 2018;186:128-37.

10 Sekhon MS, Griesdale DE, Robba C, et al. Optic nerve sheath diameter on computed tomography is correlated with simultaneously measured intracranial pressure in patients with severe traumatic brain injury. Intensive Care Med 2014;40:1267-74

11 Kimberly HH, Noble VE. Using MRI of the optic nerve sheath to detect elevated intracranial pressure. Crit Care 2008;12:181.

12 Dubourg J, Javouhey E, Geeraerts T, et al. Ultrasonography of optic nerve sheath diameter for detection of raised intracranial pressure: a systematic review and metaanalysis. Intensive Care Med 2011;37:1059-68.

13 Geeraerts T, Merceron S, Benhamou D, et al. Non-invasive assessment of intracranial pressure using ocular sonography in neurocritical care patients. Intensive Care Med 2008;34:2062-7

14 Tayal VS, Neulander M, Norton HJ, et al. Emergency department sonographic measurement of optic nerve sheath diameter to detect findings of increased intracranial pressure in adult head injury patients. Ann Emerg Med 2007;49:508-14.

15 Wang L, Feng L, Yao Y, et al. Optimal optic nerve sheath diameter threshold for the identification of elevated opening pressure on lumbar puncture in a Chinese population. PLoS One 2015;10:e0117939.

16 Padayachy LC, Padayachy V, Galal U, et al. The relationship between transorbital ultrasound measurement of the optic nerve sheath diameter (ONSD) and invasively measured ICP in children. Part II: age-related ONSD cut-off values and patency of the anterior fontanelle. Childs Nerv Syst 2016:32:1779-85.

17 Lee SU, Jeon JP, Lee H, et al. Optic nerve sheath diameter threshold by ocular ultrasonography for detection of increased intracranial pressure in Korean adult patients with brain lesions. Medicine 2016;95:e5061.

18 Wang LJ, Yao Y, Feng LS, et al. Noninvasive and quantitative intracranial pressure estimation using ultrasonographic measurement of optic nerve sheath diameter. Sci Rep 2017;7:42063

19 Toms DA. The mechanical index, ultrasound practices, and the ALARA principle. J Ultrasound Med 2006:25:560-1. 
20 Bäuerle J, Lochner P, Kaps M, et al. Intra- and interobsever reliability of sonographic assessment of the optic nerve sheath diameter in healthy adults. J Neuroimaging 2012;22:42-5.

21 Ballantyne SA, O'Neill G, Hamilton R, et al. Observer variation in the sonographic measurement of optic nerve sheath diameter in normal adults. Eur J Ultrasound 2002;15:145-9.

22 Kabat AG. Intracranial hypertension. Optom Clin 1996;5:153-79.

23 Killer HE, Laeng HR, Flammer J, et al. Architecture of arachnoid trabeculae, pillars, and septa in the subarachnoid space of the human optic nerve: anatomy and clinical considerations. Br J Ophthalmol 2003;87:777-81.

24 Dubost C, Le Gouez A, Zetlaoui PJ, et al. Increase in optic nerve sheath diameter induced by epidural blood patch: a preliminary report. $\mathrm{Br} J$ Anaesth 2011;107:627-30.

25 Launey $Y$, Nesseler N, Le Maguet P, et al. Effect of osmotherapy on optic nerve sheath diameter in patients with increased intracranial pressure. J Neurotrauma 2014;31:984-8.

26 Shah S, Kimberly H, Marill K, et al. Ultrasound techniques to measure the optic nerve sheath: is a specialized probe necessary? Med Sci Monit 2009;15:Mt63-8.
27 Wang LJ, Chen LM, Chen Y, et al. Ultrasonography assessments of optic nerve sheath diameter as a noninvasive and dynamic method of detecting changes in intracranial pressure. JAMA Ophthalmol 2018;136:250-6.

28 Nusbaum DM, Antonsen E, Bockhorst KH, et al. Optic nerve sheath diameter measurement techniques: examination using a novel ex-vivo porcine model. Aviat Space Environ Med 2014;85:50-4.

29 Helmke K, Hansen HC. Fundamentals of transorbital sonographic evaluation of optic nerve sheath expansion under intracranial hypertension. I. Experimental study. Pediatr Radiol 1996;26:701-5.

30 Helmke K, Hansen HC. Fundamentals of transorbital sonographic evaluation of optic nerve sheath expansion under intracranial hypertension II. Patient study. Pediatr Radiol 1996;26:706-10.

31 Hansen HC, Helmke K. Validation of the optic nerve sheath response to changing cerebrospinal fluid pressure: ultrasound findings during intrathecal infusion tests. J Neurosurg 1997;87:34-40.

32 Choi SH, Min KT, Park EK, et al. Ultrasonography of the optic nerve sheath to assess intracranial pressure changes after ventriculo-peritoneal shunt surgery in children with hydrocephalus: a prospective observational study. Anaesthesia 2015;70:1268-73. 